\title{
Positive Temperature Coefficient Effect of Epoxy Resin Filled with Short Carbon Fibers
}

\author{
Yuri CheKanov, ${ }^{*, * *}$ Ryuji Ohnogi, ${ }^{*}$ Shigeo Asai, ${ }^{*}$ \\ and Masao Sumita* \\ *Department of Organic and Polymeric Materials, Tokyo Institute of Technology, \\ Ookayama Meguro-ku, Tokyo 152-8552, Japan \\ ** Institute of Chemical Physics in Chernogolovka, Russian Academy of Sciences, \\ 142432, Chernogolovka, Moscow Region, Russia
}

(Received August 29, 1997)

\begin{abstract}
A phenomenon of positive temperature coefficient (PTC) of electrical resistivity has been studied for an epoxy resin filled with carbon fibers (CF). An upper limit temperature of the PTC region was found to depend strongly on CF content. The greater CF content is, the higher upper limit temperature of PTC region is observed. A phenomenological model was proposed to explain the PTC effect. Cure temperature was shown to have a great influence on upper limit temperature of PTC region and resistivity value at room temperature. The current-voltage characteristics of the composites are not Ohmic in high-voltage range, implying non-graphitic mechanism of conduction. Tunneling mechanism of the conduction is supposed. KEY WORDS Epoxy Resin / Positive Temperature Coefficient / Carbon Fibers / Electrical Resistivity Percolation /
\end{abstract}

Carbon blacks (CB) and carbon fibers (CF) are widely used for a manufacture of electrically conductive polymer composites. A variety of applications of such composites are rather wide. They are used as antistatic materials, shields, resistors ${ }^{1}$ and heaters. ${ }^{2}$ Materials possessing positive temperature coefficient (PTC) effect are one of them. PTC effect means that electrical resistivity of a material increases during heating. This effect was extensively studied mostly for thermoplastic polymers filled with carbon blacks. ${ }^{3-11}$ A maximum of resistivity of such composites was found to take place in the vicinity of polymer melting point. However, a sharp decrease of resistivity (so called NTC effect) happens at once after the maximum. Moreover, a reproducibility of PTC effect strongly deteriorates throughout successive temperature cycles. In order to avoid NTC effect and improve reproducibility a radiation crosslinking of polymer matrix was successfully used. ${ }^{6,9}$ Thus, crosslinked polymers seem to be the most attractive ones for a manufacture of PTC composites.

A mechanism of PTC effect is not fully understood. Generally speaking, the resistivity (or conductivity) of polymer composites filled with the conductive particles strongly depends on filler content and can be explained in terms of percolation theory ${ }^{12-14}$ as follows. Conductive particles agglomerate in composite as clusters. The size and number of clusters increase with increasing of filler content. At a critical content that is called a percolation threshold a cluster becomes infinite and the material becomes conductive. An apparent content of carbon filler decreases in course of heating because polymer matrix has a coefficient of thermal expansion which is two orders of magnitude higher than that of $\mathrm{CB}$ or CF. However the effective decreasing of CF content due to heating of epoxy matrix is rather small and can not explain PTC effect in some cases for high loadings of CF. ${ }^{15}$ Thus additional studies about nature of the conductivity in course of heating are necessary.

In order to keep a material conductive, a good elec- trical contact between particles is also essential. For example, if one breaks all contacts off in composite with an infinite cluster of conductive particles then the composite becomes isolative. Thus the infinite cluster of the particles and good electrical contacts between them are two necessary conditions for composite being conductive. In works of Carmona et al. a phenomenological model of PTC effect in terms of decreasing of a contact concentration during heating was proposed. ${ }^{15-17}$ The main idea of these works was that a probability of inter-fiber contact opening is increasing in course of heating and at a certain temperature all contacts are open. So, changing of temperature can be one of the factors that can lead to breaking off contacts. In the presented work an effort was made to describe PTC effect in terms of decreasing of a number of contacts during heating of the composite and also way of the contact formation while a cure schedule was considered.

\section{EXPERIMENTAL}

\section{Composite Components and Preparation}

Epoxy resin ACR R-1415 from Shin-nittetsu corporation was used in the study. The resin was a highly viscous liquid at room temperature. Glass transition temperature of fully cured epoxy resin $\left(T_{\mathrm{g} \infty}\right)$ was equal to $100 \pm 2^{\circ} \mathrm{C}$ as measured by DSC. Short carbon fibers (SCF) KUREHA M101 were used as a conductive filler. The fibers had an average length of $60 \mu \mathrm{m}$ and diameter of $16 \mu \mathrm{m}$. Carbon fibers were degassed under vacuum for $1 \mathrm{~h}$ at $120^{\circ} \mathrm{C}$ before mixing with epoxy resin. Epoxy resin was heated to $90^{\circ} \mathrm{C}$ and then mixed up thoroughly with carbon fibers for $10 \mathrm{~min}$ by hands. After mixing, the liquid composition was degassed under vacuum for $10 \mathrm{~min}$ at $90^{\circ} \mathrm{C}$ in order to remove air bubbles. Then the liquid composition was poured between glass plates separated to $1 \mathrm{~mm}$ and cured there by the following cure schedule. Three cure schedules were employed in the work: 
1. Cure at $150^{\circ} \mathrm{C}$ for $1 \mathrm{~h}$.

2. Cure at $120^{\circ} \mathrm{C}$ for $2 \mathrm{~h}$ followed by curing at $150^{\circ} \mathrm{C}$ for $1 \mathrm{~h}$.

3. Cure at $90^{\circ} \mathrm{C}$ for $2 \mathrm{~h}$ followed by curing at $150^{\circ} \mathrm{C}$ for $1 \mathrm{~h}$.

All cure schedules ensure full cure of epoxy matrix that was checked by DSC. The most of the data were obtained for composites cured by the first schedule. Other two schedules were employed only in part of the work where the influence of cure schedule on PTC effect was studied. Composite samples were sheets with thickness $1 \mathrm{~mm}$.

\section{Electrical Resistivity Measurement}

Specimens in form of square $20 \times 20 \mathrm{~mm}^{2}$ were cut from composite sheets. At least three samples with the total number of specimens of 6 were utilized to take resistivity values for each $\mathrm{CF}$ content studied. Specimen surface was covered by a silver paste in order to ensure good electrical contact with the copper electrodes. Resistivity was measured through the specimen thickness. The specimen with the attached copper electrodes was placed in a special chamber where temperature was controlled in accordance with a desirable program. Heating rate during electrical measurements was $4^{\circ} \mathrm{C} \mathrm{min}^{-1}$. A picoammeter 487 from Keithley Instruments, Inc. equipped with a voltage source was employed for the resistivity measurements. Only direct current was used to measure resistivity values. An original software was developed to measure the resistivity with temperature. The software maintained the recording of resistivity with temperature interval $1^{\circ} \mathrm{C}$. Each record lasted less than $5 \mathrm{~s}$ and involved the measurements of resistivity at several voltage values. One temperature scan produced several dependencies of resistivity on temperature at different voltage values. Voltage values were taken in a range from 0.001 to $500 \mathrm{~V}$.

The resistivity of polymers is usually time-dependent due to effect of current absorption. ${ }^{19}$ Because the resistivity was measured during short period of time it was important to evaluate the difference between an initial value of resistivity and the stationary one. The initial value of the resistivity of epoxy was approximately in two times less than the stationary one at temperatures below $T_{\mathrm{g} \infty}$. This difference was practically negligible at temperatures above $T_{\mathrm{g} \infty}$. The initial values of resistivity were used throughout this work. The resistivity of composites with CF content higher than percolation threshold did not depend on time in all temperature range investigated.

\section{RESULTS AND DISCUSSION}

\section{Percolation and PTC Behavior}

Resistivity values at room temperature versus carbon fibers content are presented in Figure 1a. With increasing of CF content the resistivity drops sharply at CF content of $6 \%$ by volume. An exact position of the percolation threshold is one of the issues discussed in literature. Some authors define the commencement of insulator to conductor transition as the percolation threshold, ${ }^{18}$ but the others consider the end of the transition as the percolation threshold. ${ }^{20,21}$ It is beyond the scope of this

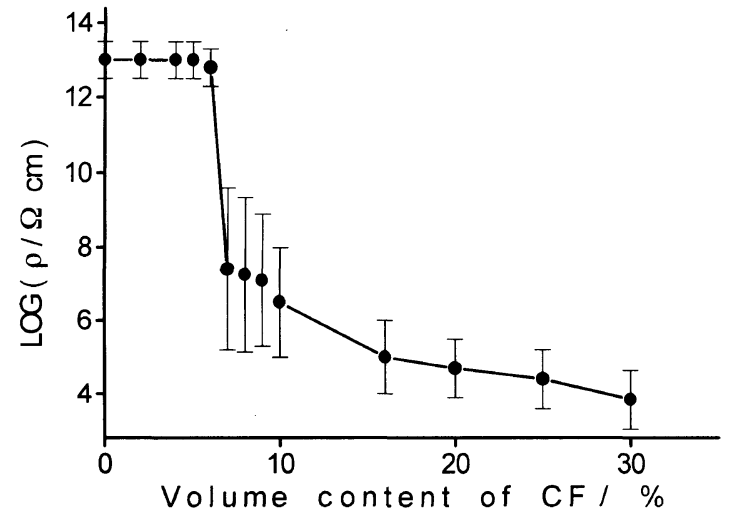

Figure 1a. Logarithm of room temperature resistivity versus volume content of carbon fibers. Resistivity values were measured at a voltage of $500 \mathrm{~V}$ for samples with CF content less than $7 \%$ and at a voltage of $1 \mathrm{~V}$ for samples with greater $\mathrm{CF}$ content.

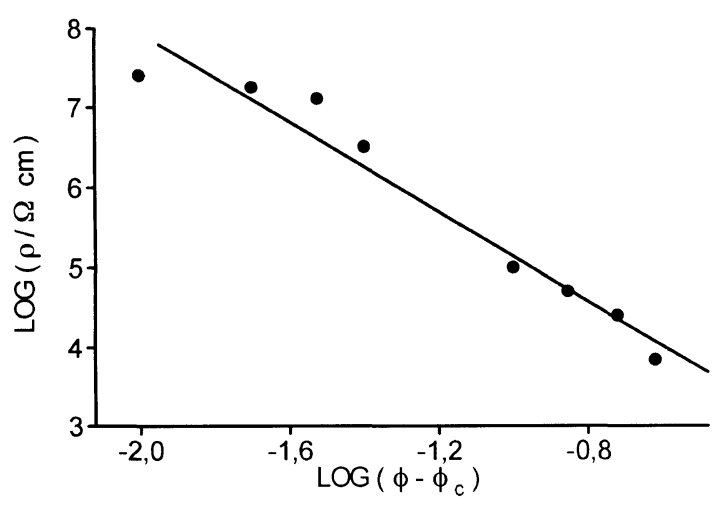

Figure 1b. Log- $\log$ plot of the resistivity against $\phi-\phi_{\mathrm{c}}$ with $\phi_{\mathrm{c}}=6 \%$ showing the power law dependence (eq 1). Straight line was plotted by least-square method and has a slope $t=2.8$.

work to discuss this question. Following Medalia, six volume percent of $\mathrm{CF}$ was defined as the percolation threshold in this work. Percolation theory predicts the dependence of composite resistivity $\rho$ upon volume content of the conductive filler as ${ }^{13}$

$$
\rho=\rho_{0}\left(\phi-\phi_{\mathrm{c}}\right)^{-t} \text { for } \phi>\phi_{\mathrm{c}}
$$

where $\rho_{0}$ is the resistivity of conductive particles, $\phi$ the volume content of filler, $\phi_{\mathrm{c}}$ the critical volume content of filler at percolation threshold, $t$ is the exponent for the dependence. From theoretical consideration $t$ should be equal to $1.5-1.6 .^{13}$ The $t$ values ranged from 1.6 to 2.0 were experimentally found for polymer-carbon black composites. $^{22,23}$ For composite filled with randomly oriented fibers $t$ value was experimentally found ${ }^{15}$ to be about 3 . For studied composites $t$ value was equal to 2.8 as shown in Figure $1 \mathrm{~b}$.

Temperature dependence of resistivity for composites with different carbon fibers content is shown in Figure 2. It is worth to note that resistivity of epoxy resin cured without CF is temperature-dependent. Below glass transition temperature it is constant and rather high. Above $T_{\mathrm{g} \infty}$, the resistivity gradually decreases with temperature. The similar dependence of resistivity on temperature was found for different epoxy resins. ${ }^{24}$ Electrical resistivity of epoxies results from migration of ions through the polymer network. The ions arise from ionic impurities and ionic products of thermal de- 


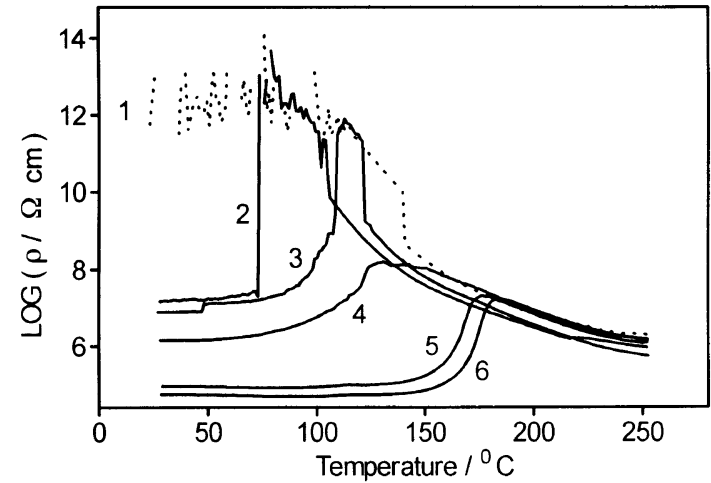

Figure 2. Dependence of electrical resistivity on temperature for the composites with different volume content of CF. 1, epoxy resin; $2,7 \%$; $3,8 \% ; 4,10 \% ; 5,16 \% ; 6,20 \%$.

gradation.

Resistivity of composites with CF content less than percolation value exhibits the same behavior as that of epoxy. Resistivity of composites with CF content higher than percolation value stays approximately constant up to a certain temperature. Then a sharp increase of resistivity occurs and resistivity reaches a maximum value. The temperature when resistivity reaches the maximum, $T_{\rho \max }$, is used in the work as an upper limit temperature of PTC region. The resistivity of composite above $T_{\rho \max }$ practically coincides with resistivity of epoxy. The $T_{\rho \max }$ value depends on $\mathrm{CF}$ content. The greater $\mathrm{CF}$ content is the higher $T_{\rho \max }$ is observed.

\section{Phenomenological Model of PTC Effect}

One of the main questions connected with PTC behavior is: "How to predict the upper limit temperature of PTC region knowing the properties of polymer matrix and those of conductive filler?" In this paragraph we propose a phenomenological model that explains the PTC behavior in our case and predicts temperature region of PTC effect for composites filled with spherical particles.

The farther examination will consider PTC effect for composites with $\mathrm{CF}$ content higher than percolation value in terms on inter-fiber contacts. Term "contact close" means that a contact is electrically conductive and term "contact open" or "broken" means that contact is not electrically conductive.

At first it is necessary to understand how contact originates. Suppose that the most of contacts occur across the fibers in composite filled with the short fibers. Thus the fiber diameter will emerge as a representative size of the filler. Suppose farther that not all fibers are in direct contact with each other in liquid composition after preparation procedure, that is, there is a gap between some of them. The inter-fiber contact is shown schematically in Figure 3. Where $l$ is the distance between fiber centers and $w$ is the gap width. Obviously, $w=l-d$, where $d$ is the fiber diameter. The change in the distances $l$ and $w$ will depend on the expansion or shrinkage of polymer matrix.

Polymer matrix shrinks in course of cure and the subsequent cooling to room temperature. Therefore, we have

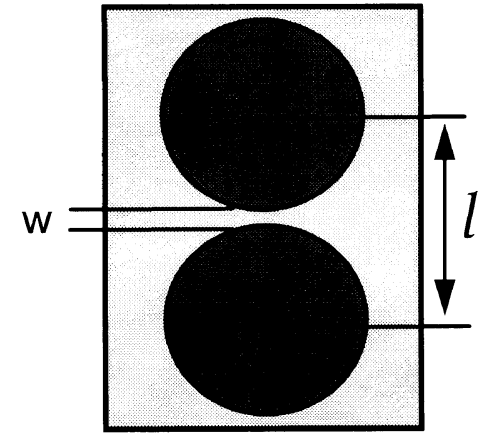

Figure 3. Composite element containing inter-fiber contact. $l$ distance between fiber centers, $w$ gap width between fibers.

$$
\begin{gathered}
\varepsilon_{\mathrm{all}}=\alpha_{\text {cure }}\left(1-X_{\text {gel }}\right) / 3+\gamma_{\mathrm{r}}\left(T_{\mathrm{g}}-T_{\text {cure }}\right)+\gamma_{\mathrm{g}}\left(T_{\text {room }}-T_{\mathrm{g}}\right), \\
\text { for } T_{\mathrm{g}}<T_{\text {cure }}
\end{gathered}
$$

where $\varepsilon_{\text {all }}$ is the linear strain of a rigid polymer matrix during cure and cooling, $\alpha_{\text {cure }}$ is the total chemical shrinkage, $X_{\mathrm{gel}}$ the degree of cure corresponding to gel-point, $\gamma_{\mathrm{g}}$ is the linear thermal expansion coefficient of epoxy resin in glassy state and $\gamma_{\mathrm{r}}$ is that in rubbery state. Thus the cure schedule will lead to a decrease in $l$. However, $l$ can not be less than $d$. After $l$ becomes $d$, the following matrix strain will be stored as a residual compressive strain. It can be expressed by next equation:

$$
\varepsilon_{\mathrm{all}}=\left(d-l_{0}\right) / l_{0}+\varepsilon_{\mathrm{res}}
$$

where $l_{0}$ is an inter-fiber distance in the liquid composition after mixing procedure, $\varepsilon_{\text {res }}$ is the residual strain of a composite element containing inter-fiber contact. The less $l_{0}$ is the greater $\varepsilon_{\text {res }}$ will be stored. For example, if $l_{0}$ is equal to $d$, fibers are in direct contact with each other than $\varepsilon_{\text {res }}=\varepsilon_{\text {all }}$. Term "direct contact" means that $w_{0}=0$, where $w_{0}=l_{0}-d$, is a gap width in the liuqid composition. If it is supposed that in the liquid composition there is a some distribution of contacts by value of $l_{0}$, then in the composite after the cure schedule will exist a some distribution of contacts by value of $\varepsilon_{\text {res }}$.

In fact, $l$ can not become $d$, since if a polymer layer existed between the fibers it could not be squeezed out because of crosslinking. However, we suppose that the layer can be strongly compressed in course of cure. That is, we suppose $w_{\text {room }} \ll w_{0}$, where $w_{\text {room }}$ is a gap width at room temperature after curing. Due to this restriction we can not evaluate $w_{\text {room }}$ which is resulted from cure schedule. However, we can evaluate the residual compressive strain and predict how the cure schedule influences the temperature region where PTC effect will occur. It is noteworthy that the proposed assumption is mostly reasonable for large filler particles, when $d \gg w_{0}$. Because even a small strain of the matrix can result in a significant strain of the polymer layer in the gap in this case.

Let us consider the contact behavior in the manufactured composite during heating. The thermal expansion of polymer matrix in the course of heating will compensate the residual strain. At some critical temperature $T_{\mathrm{c}}, \varepsilon_{\mathrm{T}}\left(T_{\mathrm{c}}\right)+\varepsilon_{\mathrm{res}}=0$ and the following heating will lead to a separation of fibers. There $\varepsilon_{\mathrm{T}}(T)$ is the linear thermal strain of polymer matrix which can be expressed as 


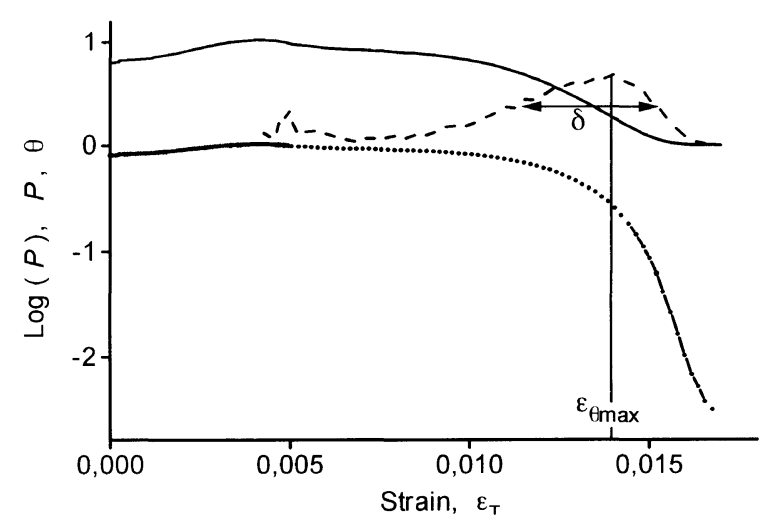

Figure 4. Dependencies of contact concentration, $P$ (solid line), $\log (P)(--)$ and $\theta$ (dashed line) on thermal strain for composite with $20 \%$ of CF. $\theta$ values were calculated as $K \cdot \mathrm{d} P / \mathrm{d} \varepsilon_{\mathrm{T}}$. Factor $K=-1 / 300$ in order to show $\theta$ values on the same scale as other data.

$\varepsilon_{\mathrm{T}}(T)=\gamma \Delta T$, in which $\gamma$ is the coefficient of linear thermal expansion of the polymer and $\Delta T$ is the temperature change from some temperature. When $\varepsilon_{\text {res }}$ is compensated by $\varepsilon_{\mathrm{T}}, \Delta T=T_{\mathrm{c}}-T_{\text {room }}$. Suppose that there is a critical value of gap width $w_{\mathrm{c}}$ such that if $w(T)>w_{\mathrm{c}}$ the contact opens. In other words, we suppose the electrical conductivity through the contact becomes equal to the polymer conductivity at $w(T)>w_{\mathrm{c}}$. It is possible to estimate $\Delta T_{\mathrm{B}}$ as a temperature change for breaking the contact by the next equation:

$$
\Delta T_{\mathrm{B}}=w_{\mathrm{c}} /(d \gamma)
$$

In this case $\Delta T_{\mathrm{B}}=T_{\mathrm{B}}-T_{\mathrm{c}}$, where $T_{\mathrm{B}}$ is temperature when the contact will be broken. Taking $w_{\mathrm{c}}=10 \mathrm{~nm},{ }^{8,18}$ $d=16 \mu \mathrm{m}$, and $\gamma=1.7 \times 10^{-4} \mathrm{~K}^{-1}$ as a typical value of linear coefficient of thermal expansion for epoxies in rubbery state, ${ }^{25,26}$ one can calculate $\Delta T_{\mathrm{B}}=3.7^{\circ} \mathrm{C}$. It is obvious that a width of temperature interval where PTC effect occurs can not be less than $\Delta T_{\mathrm{B}}$ value. More narrow temperature interval where PTC effect occurs can be expected from eq 4 for the larger filler particles.

Let us express the behavior of resistivity with temperature in terms of a change of contact concentration $P(T)$, as follows $P(T)=\rho_{\text {room }} / \rho(T) . \quad P\left(T_{\text {room }}\right)=1$ and $0<$ $P(T)<1$ because the most number of contacts exist in the composite at room temperature and the heating can only decrease the number of contacts. So, the dependence of $\log (\rho)$ on temperature can be represented as the dependence of $\log (P)$ on strain $\varepsilon_{\mathrm{T}}$. Taking derivative of $P$ with respect to $\varepsilon_{\mathrm{T}}, \theta=\mathrm{d} P / \mathrm{d} \varepsilon_{\mathrm{T}}$. It is possible to find the dependence of $\theta$ on $\varepsilon_{\mathrm{T}}$, where $\theta\left(\varepsilon_{\mathrm{T}}\right)$ is the rate of opening of the contacts at strain $\varepsilon_{\mathrm{T}}$. In Figure 4 the dependencies of $\log (P), P$, and $\theta$ upon $\varepsilon_{\mathrm{T}}(T)$ are shown. The temperature dependence of $\varepsilon_{\mathrm{T}}$ was calculated by following equations:

$$
\begin{array}{lc}
\varepsilon_{\mathrm{T}}(T)=\gamma_{\mathrm{g}}\left(T-T_{\text {room }}\right), & T \leq T_{\mathrm{g}} \\
\varepsilon_{\mathrm{T}}(T)=\gamma_{\mathrm{g}}\left(T_{\mathrm{g}}-T_{\text {room }}\right)+\gamma_{\mathrm{r}}\left(T-T_{\text {room }}\right), \quad T>T_{\mathrm{g}} \\
\gamma_{\mathrm{g}}=6.7 \times 10^{-5} \mathrm{~K}^{-1}, \quad \gamma_{\mathrm{r}}=1.7 \times 10^{-4} \mathrm{~K}^{-125,26}
\end{array}
$$

As a matter of fact because of the presence of a small negative slope on the dependencies of resistivity on temperature in the range from $T_{\text {room }}$ to $T_{\mathrm{g} \infty}$, the $\rho_{\text {min }}$ was used instead of $\rho_{\text {room }}$ as a resistivity at which $P=1$. As parameters for characterization of contact distribution

\begin{tabular}{|c|c|c|c|c|}
\hline Volume content of $\mathrm{CF}$ & & & $w_{0}$ & $T_{\rho \max }$ \\
\hline$\%$ & & & $\mathrm{~nm}$ & ${ }^{\circ} \mathrm{C}$ \\
\hline 7 & $3.3 \pm 2.2$ & $2.0 \pm 1.7$ & 257 & $91 \pm 16$ \\
\hline 8 & $3.7 \pm 0.6$ & $3.3 \pm 1.3$ & 251 & $108 \pm 9$ \\
\hline 10 & $4.3 \pm 1.0$ & $2.7 \pm 1.0$ & 241 & $118 \pm 8$ \\
\hline 16 & $14.0 \pm 0.9$ & $4.3 \pm 0.7$ & 82 & $173 \pm 8$ \\
\hline 20 & $13.3 \pm 0.7$ & $4.0 \pm 0.3$ & 94 & $170 \pm$ \\
\hline 25 & $14.7 \pm 0.8$ & $4.0 \pm 0.5$ & 71 & $180 \pm$ \\
\hline 30 & $13.8 \pm 0.9$ & $4.0 \pm 0.4$ & 86 & $178 \pm 7$ \\
\hline
\end{tabular}

Table I. Values $\varepsilon_{\theta_{\max }}$ and $\delta$ calculated from experimental data for composites with different $\mathrm{CF}$ content. Initial gap, $w_{0}$, was calculated by using eq 6 as $w_{0}=l_{0}-d$

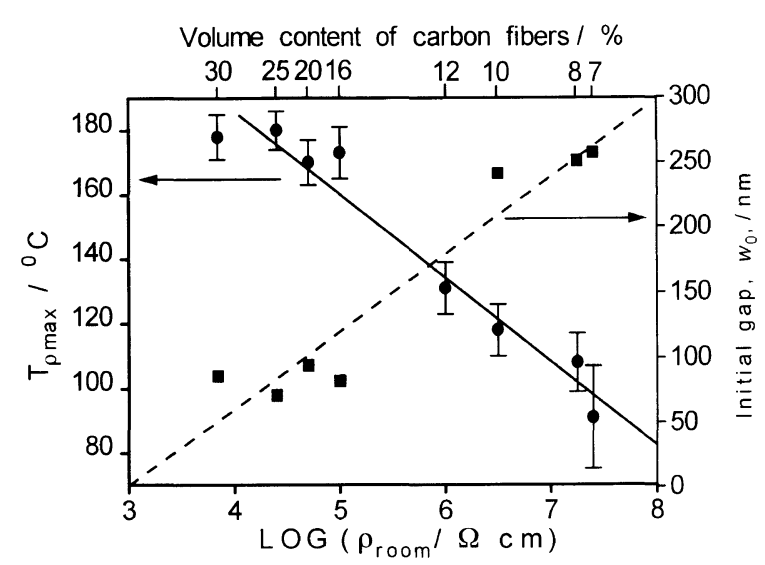

Figure 5. Temperature corresponding to maximum of resistivity, $T_{\rho \max }$, and initial gap in the liquid composition $w_{0}$ versus logarithm of composite resistivity at room temperature. Volume content of carbon fibers is shown on the top scale as an aid.

the values of $\varepsilon_{\theta \max }$ and $\delta$ were used. $\varepsilon_{\theta \max }$ is a strain magnitude when the most number of contacts are opening. $\delta$ is a width of $\theta\left(\varepsilon_{\mathrm{T}}\right)$ distribution taken on half of $\theta_{\max }$ altitude. Taking into account that for contact opening firstly the $\varepsilon_{\text {res }}$ compensation and secondly the some additional heating $\Delta T_{\mathrm{B}}$ (see eq 4 ) are necessary, the next equation can be written

$$
\varepsilon_{\theta \max }+\varepsilon_{\mathrm{res}}-w_{\mathrm{c}} / d=0
$$

Substituting $\varepsilon_{\text {res }}$ value from eq 7 into eq 3 it is possible to estimate the $l_{0}$ value as follows:

$$
l_{0}=d /\left(1+\varepsilon_{\mathrm{a} 11}+\varepsilon_{\theta \max }-w_{\mathrm{c}} / d\right)
$$

If $\varepsilon_{\text {all }}$ is supposed to be approximately equal to $-1.8 \times 10^{-2}$ in assumption that $\alpha_{\text {cure }}=-0.06$ and $X_{\text {gel }}=0.75^{27}$ and $\varepsilon_{\theta \max }$ values are taken from experimental data like on Figure 4 , it is possible to find $l_{0}$ and $w_{0}$ values for different contents of carbon fibers. These results are presented in Table I. It can be seen from the Table I that the gap between fibers in the liquid composition is smaller for higher CF content. It was found that the dependencies of $w_{0}$ and $T_{\rho \max }$ on $\log (\rho)$ are approximately linear (Figure 5). Following to the above estimations, we were able to find the dependence of the gap width upon $\mathrm{CF}$ content. If one will know the dependence of $w_{0}$ upon $\mathrm{CF}$ content a priori, it will be possible to predict temperature region of PTC effect. For spherical filler particles, for example, there is a theoretical dependence of distance between particles upon their content for cubic packing ${ }^{28}$ : 


$$
w_{0}=d(\sqrt[3]{\pi /(6 \phi)}-1)
$$

Thus using eq 8 and 9 one can calculate for a composite with spherical filler particles:

$$
\varepsilon_{\theta \max }=w_{\mathrm{c}} / d+\sqrt[3]{6 \phi / \pi}-1-\varepsilon_{\mathrm{all}}
$$

and knowing thermal expansion coefficient of polymer, one can predict temperture region of PTC effect. However, in order to reproduce the dependence of resistivity on temperature completely, it is necessary to know the distribution of contacts by value of $w_{0}$ in composite in order to predict the dependence of $\theta$ upon $\varepsilon_{\mathrm{T}}(T)$ as shown in Figure 4.

The presented model is based mainly on the consideration of the contact formation and might be a part of a more general model. In our opinion, this general model should take into account the following factors in order to predict the behavior of resistivity with temperature. (i) To estimate the absolute number of contacts and their distribution by gap width in liquid composition; mixing procedure, filler content and shape seem to be important for this estimation. (ii) To describe the contact behavior, namely gap width and residual strain, in the course of cure; by using the model proposed in the paper it is possible to evaluate only the residual strain stored in the place of the contact after curing. In order to evaluate the gap width, a calculation of a strain distribution in the place of the contact is necessary. Knowledge of the gap width resulted from cure schedule will allow to predict current-voltage characteristics. (iii) To evaluate the fraction of the filler which is involved in the infinite conductive cluster with using of the percolation theory; obviously, the value of the fraction will depend on both content of the filler and ambient conditions. For instance, heating will lead to increasing of the gap width and breaking off contacts, because of this the fraction of the conductive particles participating in the infinite cluster will decrease.

\section{Influence of Cure Schedule on PTC Behavior}

The epoxy resin containing $20 \%$ by volume of CF was cured by three above mentioned schedules. The dependencies of the logarithm of resistivity on temperature are plotted in Figure 6 for these composites. The cure schedule is shown to affect both $T_{\rho \max }$ and the initial value of resistivity at room temperature. Let us discuss the influence on $T_{\rho \max }$ at first.

The main difference in the schedules is the value of cure temperature $T_{\text {cure }}$ on the first step of cure. Epoxy resin reaches gel-point on the first step of cure and transforms from liquid to rubber. Material remembers the temperature when it becomes rigid and because of this strain value $\varepsilon_{\text {all }}$ will depend on cure schedule. The first term in right hand side of eq 2 is the same for all cure schedules because full cure takes place in all cases. Total value of two other terms is determined by $T_{\text {cure }}$ value. For instance, the second term in eq 2 will disappear for the third cure schedule since in this case $T_{\text {cure }}<T_{\mathrm{g}}$. It is worth to note that thermal strain at heating from $T_{\text {cure }}$ to $150^{\circ} \mathrm{C}$ for the second and third cure schedules will be compensated by strain at final cooling. Thus only difference between $T_{\text {cure }}$ and $T_{\text {room }}$ makes a contribution

Polym. J., Vol. 30, No. 5, 1998

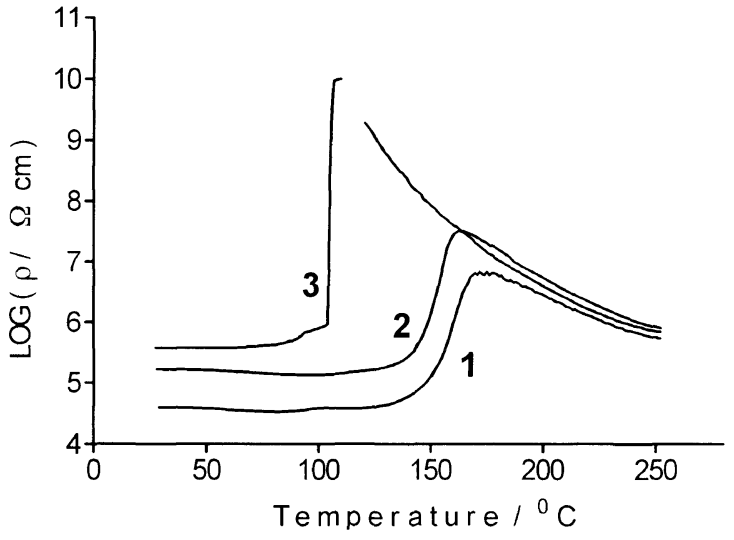

Figure 6. Temperature dependencies of the logarithm of the resistivity for the composite with $20 \%$ of $\mathrm{CF}$ cured by different schedules (see the text). Values of resistivity were measured at $1 \mathrm{~V}$ for the curve 1 and $30 \mathrm{~V}$ for the curves 2 and 3 . Digits near curves correspond to the cure schedule number.

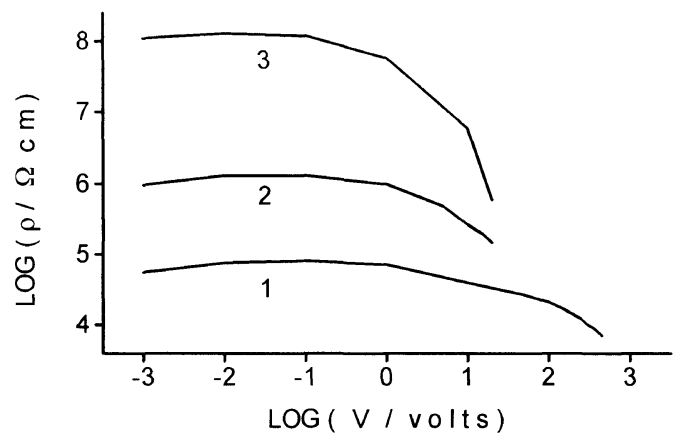

Figure 7. Dependence of room temperature resistivity on voltage on a $\log -\log$ scale for composite with $20 \%$ of CF cured by different schedules. Digits near curves correspond to the cure schedule number.

in $\varepsilon_{\text {all }}$ value. In eq 3 different values of $\varepsilon_{\text {all }}$ will lead to different values of residual strain and consequently to different upper limit temperatures of PTC region. As a rough estimation it is possible to propose basing on the model that the difference in $T_{\rho \max }$ should be the same as the difference in $T_{\text {cure }}$. This is the case when one compares the first and the third cure schedules, but for the second cure schedule $T_{\rho \max }$ is on $20^{\circ} \mathrm{C}$ degree higher than can be expected.

Let us consider the way of contact formation in more detail before discussing the influence of cure schedule on resistivity value at room temperature. It was assumed in the previous consideration that the fibers are coming into direct contact with each other during cure schedule. However, it is a rough assumption. The polymer layer between fibers can not be squeezed out if matrix is not in the liquid state. Thus polymer gap contracts in the course of cure schedule but does not disappear. This supposition relates to some mean value of the gap width not equal zero. The gap width after cure schedule depends both on residual strain value and deformative properties of the polymer. Therefore cure schedule influences the residual strain value and consequently the polymer gap width between fibers.

In the case of presence of gap between fibers, the conduction mechanism should not be graphitic when the current flows only through fibers. The current-voltage characteristic of a material obeys Ohm's law if graphitic 
Table II. Calculated values of the gap width between carbon fibers at room temperature for composites with $20 \%$ of CF cured by different schedules. Values of resistance, $R$, were taken from Ohmic region at $0.1 \mathrm{~V}$

\begin{tabular}{cccc}
\hline Schedule & $\begin{array}{c}\text { Logarithm of } \\
\text { resistance, } \\
\log (R / \Omega)\end{array}$ & $\begin{array}{c}\text { Logarithm of } \\
\text { resistivity, } \\
\log (\rho / \Omega \mathrm{cm})\end{array}$ & Gap width, $w_{\text {room }}$ \\
\hline 1 & 3.6 & 4.9 & $\mathrm{~nm}$ \\
2 & 4.8 & 6.1 & 8.1 \\
3 & 6.8 & 8.1 & 8.6 \\
\hline
\end{tabular}

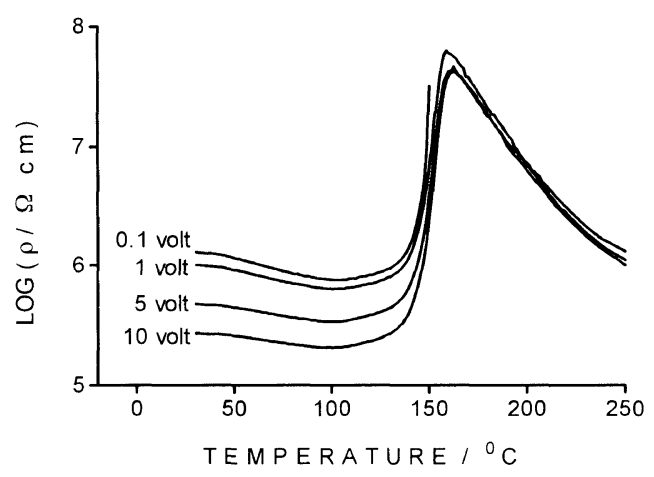

Figure 8. Dependence of the logarithm of resistivity on temperature at different values of voltage. Composite with $20 \%$ of CF cured by the second schedule.

type of conduction takes place. ${ }^{18}$ The dependence of the logarithm of resistivity for composites cured by different schedules upon voltage is presented in Figure 7. Resistance $R$ and resistivity values have to be independent of voltage if the conduction obeys Ohm's law. As one can see from Figure 7, Ohmic behavior exists only at small voltages less than $0.1 \mathrm{~V}$, so the mechanism of conduction is not graphitic. Another possible mechanism is a tunneling of electrons through a polymer gap.

Simmons $^{29}$ has derived a generalized equation of the current-voltage characteristic of the electric tunnel effect between two electrodes separated by a thin insulating film. As it was shown in the paper, there is a linear relation between current and voltage in the low voltage range.

$$
I=\frac{3 \sqrt{2 m \beta}}{2 w}\left(\frac{e}{h}\right)^{2} V^{*} \exp \left\{-\frac{4 \pi w}{h} \sqrt{2 m \beta}\right\}
$$

where $m$ and $e$ are an electron mass and charge respectively, $h$ Planck's constant, $I$ the current, $V$ the voltage, $w$ the thickness of insulating film, $\beta$ the height of the potential barrier between the electrodes which was taken equal to $0.2 \mathrm{eV}^{30}$ in following calculations. Although this equation was derived for tunnel junction on microscale we suppose that the ratio of macroscopic voltage to current can be used in order to estimate the film thickness at room temperature $w_{\text {room }}$. Substituting the resistance value instead of $V / I$ in eq 11 the values of $w_{\text {room }}$ were calculated (see Table II). Because the approximate value of $\beta$ was used in the calculations, the value of the calculated gap width can also be only approximate one. However, one can see from the comparison of the calculated results how the cure schedule influences the gap width.
Resistivity at room temperature strongly depends on voltage as shown in Figure 7. This voltage dependence of resistivity is also observed throughout heating (Figure 8 ). This resistivity behavior in Figure 8 can be explained as follows. There is a gap between the fibers at room temperature and a residual compressive strain is also stored in the place of the contact. The gap width remains practically constant during heating so long as thermal strain is compensating the residual one. As it proceeds, the fibers begin to separate and when the gap width exceeds a critical value, composite resistivity became the same as a polymer resistivity.

There is a small negative slope of the resistivity behavior in Figure 8 before PTC region. The most possible reason of this NTC effect, in our opinion, is a decreasing of $\mathrm{CF}$ resistivity with heating. ${ }^{16}$

\section{CONCLUSIONS}

PTC effect of the epoxy resin filled with short carbon fibers is studied. The main reason of PTC was supposed to be a breaking off of inter-fiber contacts. In the composite with higher content of CF, there is smaller inter-fiber distance and because of this the greater compressive strain is stored during cure schedule. Therefore the heating to higher temperature is needed to break off all contacts in the composite. The upper limit temperature of PTC region was found to depend linearly on the logarithm of room temperature resistivity.

Cure schedule has an influence both on upper limit temperature of PTC region and resistivity value at room temperature. The less cure temperature is the smaller $T_{\rho \max }$ and greater value of the resistivity are observed. Current-voltage characteristics of the composites are not Ohmic in the high voltage range implying non-graphitic mechanism of conduction. Tunneling mechanism of conduction is supposed.

Acknowledgments. One of us (Yu. C.) wishes to thank Tokyo Institute of Technology for support of his work at the Institute as a visiting researcher.

\section{REFERENCES}

1. S. Nakamura, A. Ito, and G. Sawa, Polym. J., 22, 502 (1990).

2. H. H. Hassan, E. M. Abdel-Bary, and M. Amin, J. Appl. Polym. Sci., 39, 1903 (1990).

3. F. Bueche, J. Appl. Phys., 44, 532 (1973).

4. J. Meyer, Polym. Eng. Sci., 13, 462 (1973).

5. C. Klason and J. Kubat, J. Appl. Polym. Sci., 19, 831 (1975).

6. M. Narkis, A. Ram, and Z. Stain, Polym. Eng. Sci., 21, 1049 (1981).

7. K. T. Chung, A. Sabo, and A. P. Pica, J. Appl. Phys., 53, 6867 (1982).

8. R. D. Sherman, L. M. Middleman, and S. M. Jacobs, Polym. Eng. Sci., 23, 36 (1983).

9. H. Tang, J. Piao, X. Chen, Y. Luo, and S. Li, J. Appl. Polym. Sci., 48, 1795 (1993).

10. W. Jia and X. Chen, J. Appl. Polym. Sci., 54, 1219 (1994).

11. Y. Agari, A. Ueda, and S. Nagai, J. Appl. Polym. Sci., 52, 1223 (1994).

12. R. Zallen, in "The Physics of Amorphous Solids," Wiley, New York, N.Y., 1983, Chapter 4.

13. S. Kirkpatrick, Rev. Mod. Phys., 45, 574 (1973).

14. F. Lux, J. Mater. Sci., 28, 285 (1993).

15. F. Carmona, E. Valot, and L. Servant, J. de Physique I, General Physics, 2, 503 (1992). 
16. F. Carmona and C. Mouney, J. Mater. Sci., 27, 1322 (1992).

17. F. Carmona and E. Valot, Abstacts of Papers, International Symposium on Carbon (Tsukuba), 1990, p 462.

18. A. I. Medalia, Rubber Chem. Technol., 59, 432 (1986).

19. A. Fukuda, in "Measurement Methods for Fibers and Polymers," Asakura Syoten Publishing Co., Ltd., Tokyo, 1985, Chapter $5-10$, p 250.

20. Y. Song, T. W. Noh, S. I. Lee, and J. R. Gaines, Phys. Rev., 33, 904 (1986).

21. I. Balberg, Phys. Rev. Lett., 59, 1305 (1987).

22. J. Yacubowicz and M. Narkis, Polym. Eng. Sci., 26, 1568 (1986).

23. J. Yacubowicz, M. Narkis, and L. Benguigui, Polym. Eng. Sci.,
28, 1581 (1988).

24. R. W. Warfield and M. C. Petree, Polymer, 1, 178 (1960).

25. J. Kulawik, Z. Szeglowski, T. Czapla, and J. P. Kulawik, Colloid Polym. Sci., 267, 970 (1989).

26. H. Lee and K. Nevill, "Handbook of Epoxy Resin," McGraw-Hill, New York, N.Y., 1982.

27. Y. A. Chekanov, V. N. Korotkov, E. A. Dhzavadyan, L. M. Bogdanova, and B. A. Rozenberg, Polymer, 36, 2013 (1995).

28. S. Wu, Polymer, 26, 1855 (1985).

29. J. G. Simmons, J. Appl. Phys., 34, 1793 (1963).

30. P. Sheng, E. K. Sichel, and J. I. Gittleman, Phys. Rev. Lett., 40, 1197 (1978). 